Original paper

\title{
On the evaluation of edgeless diode detectors for patient-specific QA in high-dose stereotactic radiosurgery
}

\author{
Elena De Martin ${ }^{\mathrm{a}, *}$, Sultan Alhujaili ${ }^{\mathrm{b}, \mathrm{f}}$, Maria Luisa Fumagalli ${ }^{\mathrm{a}}$, Francesco Ghielmetti ${ }^{\mathrm{a}}$, \\ Marcello Marchetti ${ }^{\mathrm{c}}$, Pasqualina Gallo ${ }^{\mathrm{a}}$, Domenico Aquino ${ }^{\mathrm{d}}$, Francesco Padelli ${ }^{\mathrm{d}}$, \\ Jeremy Davis $^{\mathrm{b}}$, Saree Alnaghy ${ }^{\mathrm{b}}$, Mauro Carrara ${ }^{\mathrm{e}}$, Laura Fariselli ${ }^{\mathrm{c}}$, Anatoly B. Rosenfeld ${ }^{\mathrm{b}}$, \\ Marco Petasecca ${ }^{\text {b }}$

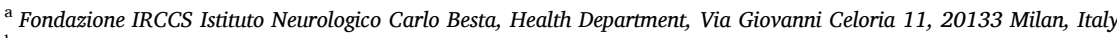 \\ ${ }^{\mathrm{b}}$ Centre for Medical Radiation Physics, University of Wollongong, Northfields Avenue, Wollongong, NSW 2522, Australia \\ ${ }^{\mathrm{c}}$ Fondazione IRCCS Istituto Neurologico Carlo Besta, Radiotherapy Unit, Department of Neurosurgery, Via Giovanni Celoria 11, 20133 Milan, Italy \\ ${ }^{\mathrm{d}}$ Fondazione IRCCS Istituto Neurologico Carlo Besta, Neuroradiology Unit, Via Giovanni Celoria 11, 20133 Milan, Italy \\ ${ }^{\mathrm{e}}$ Fondazione IRCCS Istituto Nazionale Dei Tumori, s.s.d. di Fisica Medica, Dipartimento di Diagnostica per Immagini e Radioterapia, Via Giacomo Venezian 1, 20133 \\ Milan, Italy \\ ${ }^{\mathrm{f}}$ Medical Imaging Department, College of Applied Medical Sciences, Aljouf University, Aljouf 72388, Saudi Arabia
}

\section{A R T I C L E I N F O}

\section{Keywords:}

Silicon diode detector

Patient-specific quality assurance

Angular dependence

Stereotactic radiosurgery quality assurance

\begin{abstract}
A B S T R A C T
Purpose: In this work, the potential of an innovative "edgeless" silicon diode was evaluated as a response to the still unmet need of a reliable tool for plan dosimetry verification of very high dose, non-coplanar, patient-specific radiosurgery treatments. In order to prove the effectiveness of the proposed technology, we focused on radiosurgical treatments for functional disease like tremor or pain.

Methods: The edgeless diodes response has been validated with respect to clinical practice standard detectors by reproducing the reference dosimetry data adopted for the Treatment Planning System. In order to evaluate the potential for radiosurgery patient-specific treatment plan verification, the anthropomorphic phantom Alderson RANDO has been adopted along with three edgeless sensors, one placed in the centre of the Planning Target Volume, one superiorly and one inferiorly.

Results: The reference dosimetry data obtained from the edgeless detectors are within $2.6 \%$ for output factor, offaxis ratio and well within $2 \%$ for tissue phantom ratio when compared to PTW 60,018 diode. The edgeless detectors measure a dose discrepancy of approximately $3.6 \%$ from the mean value calculated by the TPS. Larger discrepancies are obtained in very steep gradient dose regions when the sensors are placed outside the PTV. Conclusions: The angular independent edgeless diode is proposed as an innovative dosimeter for patient quality assurance of brain functional disorders and other radiosurgery treatments. The comparison of the diode measurements with TPS calculations confirms that edgeless diodes are suitable candidates for patient-specific dosimetric verification in very high dose ranges delivered by non-isocentric stereotactic radiosurgery modalities.
\end{abstract}

\section{Introduction}

Stereotactic radiosurgery (SRS) is a radiotherapy technique that delivers precisely-targeted ionizing radiation in few high dose sessions. It is becoming an essential tool in the management of a growing number of malignant and benign brain conditions [1]. The growing number of indications has encouraged its introduction beyond a few specialized cancer care centers. However, the accuracy of dose administration in such treatments is of paramount importance to guarantee both the clinical outcome and the absence of severe toxicities, especially if the

\footnotetext{
* Corresponding author at: Fondazione IRCCS Istituto Neurologico Carlo Besta, Health Department, Via Giovanni Celoria 11, 20133 Milan, Italy.

E-mail addresses: elena.demartin@istituto-besta.it (E. De Martin), sfa744@uowmail.edu.au (S. Alhujaili), marialuisa.fumagalli@istituto-besta.it (M.L. Fumagalli),

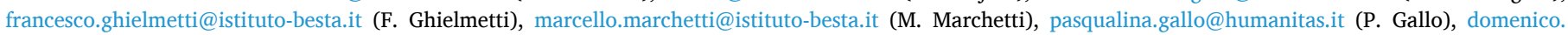

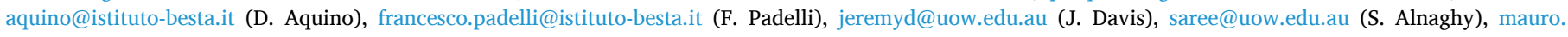
carrara@istitutotumori.mi.it (M. Carrara), laura.fariselli@istituto-besta.it (L. Fariselli), anatoly@uow.edu.au (A.B. Rosenfeld).
} 
anatomic targets include trigeminal nerve and other ganglia (for facial pain syndromes), the thalamus (for tremor or pain), the hypothalamus (for pain) and the hippocampus (for epilepsy) [2].

The total therapeutic radiation dose for these conditions varies depending on the functional treatment and fractionation protocol with dose ranges from 70 up to $160 \mathrm{~Gy}$ in single fraction [1-3]. Moreover, narrow radiation fields are used to achieve a conformal dose distribution to the target and they require exceptional care in quality assurance (QA) assessment, including patient positioning and accuracy of the dose delivered. As Codes of Practice have outlined, the challenges posed by small field dosimetry are beam-related, owing to partial occlusion of the primary source and lack of charged particle equilibrium on the beam central axis [4-7]. Complexity in small field dosimetry comes also from detector-related issues such as the detector dimensions with respect to the radiation field and the perturbation it introduces on photon and secondary electron energy fluence.

Gamma Knife ${ }^{\circledR}$ and CyberKnife ${ }^{\circledR}$ are the most common machines adopted to perform SRS. This work focuses on verification of dose distribution performed using CyberKnife ${ }^{\circledR}$ which delivers non-isocentric non-coplanar $6 \mathrm{MV}$ flattening filter-free photon beams to fixed or moving targets aligned and tracked with the aim of a stereoscopic planar imaging system [8].

The ideal dosimeter, which could be used for routine and patient specific QA in CyberKnife ${ }^{\circledR}$ SRS, would be water-equivalent at MV energies, with a sufficiently small sensitive volume to minimize volumeaveraging effects and with negligible energy and instantaneous dose rate dependence [9]. For patient-specific QA, the directional dependence of the dosimeter's response also plays a fundamental role. Additionally, it should have a wide dynamic range, readout in real-time and be easy to position in anthropomorphic or commercial phantoms that are suitable for patient-specific QA. Since commercially available devices do not satisfy all of the above criteria, it is common and recommended that different detectors are used to cross-check the consistency of results $[4,10]$. For CyberKnife $\mathbb{R}$, it is advised that QA should be performed using Gafchromic film, which has negligible directionality [3].

Gafchromic film such as EBT3 (Ashland ISP Inc., Wayne, NJ, USA) offer a 2D high spatial resolution, near tissue equivalence, weak energy and dose-rate dependence. Their potential for assessing complex dose distributions in high-precision conformal radiotherapy has been demonstrated [11-13]. However, their response is non-linear with a limited reproducibility for low doses; their use is cumbersome and requires long development times after irradiation and the definition of an established readout protocol for consistent dose readings [12,13]. Furthermore, they are recommended for use in the range of $0.01 \mathrm{~Gy}$ to $30 \mathrm{~Gy}$, inadequate to measure brain functional treatment doses [13]. Rescaling of monitor units is the common practice and consists of reducing the dose to a value within the sensitive range of film. However, this approach poses problems in the case that the minimum number of monitor units needed for the linac to achieve a stable output during irradiation is unreachable or small monitor unit (MU) beamlets are cut off by the MU threshold filtering [14].

Recently, an EBT-XD film with a sensitive range of up to $40 \mathrm{~Gy}$ was proposed [13]. Investigations have shown agreement to within $1.0 \mathrm{~mm}$ between TPS-calculated and film-measured isodoses in the range of 2 to $24 \mathrm{~Gy}$, for a $25 \mathrm{~Gy}$ maximum point dose [15-18].

Solid-state detectors, recommended for small-field dosimetry by the IAEA-AAPM code of practice, provide a range of advantages in SRS: a stable and nearly energy-independent response in megavoltage photon beams, high sensitivity and small sensitive volumes [19]. Their potential is to outperform Gafchromic films due to a wider sensitivity and dynamic range and real-time read-out. However, they require several correction factors to relate their readings to dose.

Furthermore, solid state commercial devices are angular and dose rate dependent [20]. Being non-tissue-equivalent, their response is depth and field-size dependent owing to perturbations to electron and photon fluence spectra introduced by their sensitive volumes and the extra-cameral packaging materials $[21,22]$. An extensive literature review of the angular dependence of commercial solid-state devices is reported in [23].

Correction factors that account for this effect are detector-design, linac head-design, beam quality, field size and measurement conditions dependent [4]. Thus, the design of a 'correction-free' detector would be advisable [22]. To provide $2 \mathrm{D}$ dose mapping, solid-state detectors can be arranged in detector arrays with a sufficiently low pitch in order to resolve the steep dose gradients typical of small radiation fields adopted in SRS. Still, their combined angular-dependent response would restrict their attractiveness in the case of non-isocentric deliveries.

The aim of this study was to investigate the use of a silicon diode whose response has been proven to be angular independent, for verification of machine QA and patient-specific brain functional treatment (e. g., trigeminal neuralgia, tremors, pain) plans, since they condense all the critical issues of radiosurgery treatments dosimetry: very high doses (70-160 Gy) in a single fraction were delivered using CyberKnife ${ }^{\circledR}$ by non-isocentric non-coplanar radiation fields to a target of 4 to $5 \mathrm{~mm}$ in diameter.

\section{Materials and methods}

\section{The edgeless detector}

The edgeless detector is an angular-independent single diode [24], manufactured on a thin $(100 \mu \mathrm{m})$ silicon p-type substrate. The p-n junction is realized by a 3D micro-electro mechanical (MEMS) technique with a geometry of the p-n junctions allowing an angular-independent response to within $2 \%$ for all angles between 0 and $360^{\circ}$ in both transversal and longitudinal planes. The diode has a $0.5 \times 0.5 \mathrm{~mm}^{2}$ sensitive area with no residual inactive edges.

The edgeless technology and angular dependence characterisation in MV photon beam applications is described in detail in [24]. The samples used in this study ( 3 diodes out of more than 100 available) were used in photovoltaic mode (no bias applied) and assembled using the "drop-in" technology developed by the Centre for Medical Radiation Physics (University of Wollongong, NSW, Australia) using low- $Z$ materials to minimize attenuation and perturbation of the radiation field for accurate small field dosimetry [25].

The read-out system is a custom-designed multichannel data acquisition system (DAQ) [26] based on a commercially available multichannel charge-to-frequency converter front-end called TERA (Tera Foundation, Turin, Italy) [27]. The DAQ readouts the charge generated by all the diodes simultaneously with a sampling rate of $10 \mathrm{~Hz}$ and no dead time.

\section{Dose rate dependence}

Dose rate dependence of silicon detectors used in medical linac is a well-known issue of diodes used for dosimetry [28]. It is generated by the presence of bandgap defects generated by the fabrication process and the quality of the substrate adopted. Dose per pulse (DPP) is a measure of the dose rate dependence and is particularly relevant in devices manufactured on high resistivity substrates such as the one used for the edgeless detectors [29]. DPP is measured as ratio of the diode's response to the response of a Farmer ion chamber (type 30013-PTW) in the same irradiation conditions. This method assumes that the IC is insensitive to dose rate variations which is confirmed by several studies [30]. The dose rate is varied by altering the source to surface distance (SSD) without using any attenuating material such as lead, solid water or wedges. The DPP dependence measurements were performed in the range between $0.9 \times 10^{-5}$ and $1 \times 10^{-3} \mathrm{~Gy} /$ pulse and normalised to reference conditions used for calibration at $7.8 \times 10^{-4} \mathrm{~Gy} / \mathrm{pulse}$. The variation of the instantaneous dose rate was acquired by changing SSD from 80 up to $350 \mathrm{~cm}$ with nominal dose rate of $600 \mathrm{MU} / \mathrm{min}$ on a FFF 
TrueBeam Varian linac. Error bars are calculated as one standard deviation of 5 repetitive measurements.

\section{Linearity and calibration}

Dose linearity of the edgeless diodes were carried out under reference calibration conditions. The detectors were placed inside a Solid Water phantom (Sun Nuclear, Melbourne, FL) at $15 \mathrm{~mm}$ depth with source-to-axis distance (SAD) of $800 \mathrm{~mm}$, the CyberKnife ${ }^{\circledR}$ head equipped with fixed cone of $60 \mathrm{~mm}$ diameter was positioned perpendicularly to the detectors plane. The detectors were calibrated by irradiating each diode in increments of $73.6 \mathrm{cGy}$ up to a total accumulated dose of approximately $400 \mathrm{cGy}$. Although a maximum calibration point of $400 \mathrm{cGy}$ seems to be low in respect to the total maximum irradiation dose of 200 Gy delivered for the patient plans verification, the edgeless diodes are sensitive to the dose rate and not to the total accumulated dose, making irrelevant to use very high doses to perform the calibration procedure. Each irradiation step was repeated five times to evaluate the repeatability of the measurement and the error bars reported correspond to 2 standard deviations.

Reproducing the beam parameters measured by the reference dosimeter used for the treatment planning system commissioning

We used the edgeless detectors to measure the beam parameters such as output factor, tissue-maximum ratio, off-axis ratios and dose linearity to be assured that they can replicate the relative dosimetry data used for commissioning the TPS. The data were compared to the measurements obtained by the stereotactic diode PTW 60018 (PTW, Freiburg, Germany). The measurements of edgeless detector were performed according to the methods proposed in the previous works for evaluation of their performance for small field dosimetry [24].

\section{Output factors}

Output factors (OFs) were measured by the diodes in a solid water phantom at $15 \mathrm{~mm}$ depth and $800 \mathrm{~mm}$ SAD. A dose of $200 \mathrm{MU}$ at a rate of $800 \mathrm{MU} / \mathrm{min}$ was delivered in all cases.

OFs were measured for circular fields collimated with fixed cones with $5,7.5,10,15,20,25,30,35,50$, and $60 \mathrm{~mm}$ diameters. No correction factors were applied to edgeless diode measurements.

The detector was aligned on the central axis (CAX) of the machine by maximising the response of the detector through lateral movements with $0.1 \mathrm{~mm}$ steps using a dual axis linear stager. In order to avoid any effect on OF due to misplacement of the robotic linac head, the alignment procedure of the detector in respect to the beam was repeated every time the collimator was changed.

Data collected by the edgeless diode were cross-checked with those obtained by the PTW 60018 diode used for reference dosimetry and scaled by the correction factors suggested by Francescon et al. [31]. Measurements were repeated three times to estimate uncertainty and reproducibility of the detector response.

\section{Off-axis ratios}

Off-axis ratios (OARs) were measured by the edgeless diode in a solid water phantom placed onto a dual axis stepper motor stager. The alignment of the detector was performed according with the procedure adopted for OFs. OARs were measured for circular field sizes of diameters 5, 10, 30, and $60 \mathrm{~mm}$ at $800 \mathrm{~mm} \mathrm{SAD}$ and depth of $15 \mathrm{~mm}$.

\section{Tissue phantom ratios}

Tissue phantom ratios (TPRs) were measured by the edgeless diode in a motorized water tank MP3-XS from PTW. The Edgeless diode was attached to a plastic holder, with measurements taken at $0.1 \mathrm{~mm}$ steps. TPRs were measured at various depths in the range between 0 and $20 \mathrm{~cm}$ for field sizes of 30 and $60 \mathrm{~mm}$ in diameter. For this set of measurements, the diodes were attached to the "bird cage" (provided by Accuray Inc.,
US), which keeps the SAD constant and aligns the sensors at the center of the radiation field using an inline laser pointer. The response of the detectors at each depth is normalized to the measurement taken at 15 $\mathrm{mm}$ depth. Each measurement was performed with a dose of $200 \mathrm{MU}$ at SAD of $800 \mathrm{~mm}$ and repeated 3 times to estimate uncertainty and reproducibility of the detector response. The same apparatus was used to cross-check the edgeless diode by a PTW 60018 stereotactic diode corrected according to Francescon et al. [31].

\subsection{Functional neurological disorder treatment verification: Phantom setup and treatment planning system}

Three independently calibrated diodes inserted in the Alderson RANDO anthropomorphic phantom (Imaging Solutions, Australia) were used in this experiment. In order to have an accurate localization of the sensors and to determine the doses calculated in such positions by the treatment planning system, the phantom with the diodes inserted has been scanned by a Philips Brilliance CT Simulator (Philips Electronics N. V., Netherlands). The protocol adopted is based on the clinical parameters used for acquiring intracranial image studies ( $1 \mathrm{~mm}$ slice thickness, contiguous slices, $120 \mathrm{kVp}, 400 \mathrm{mAs}$ ). No fiducials were used because the RANDO phantom has embedded bony structures which are utilised by the CyberKnife ${ }^{\circledR}$ tracking system (6DSkull, Accuray Inc., US) as reference for alignment with the machine coordinate frame. In this study the RANDO phantom has been employed in two different verification scenarios. In one a treatment plan has been optimized directly on the phan'tom by identifying a functional lesion located at the central diode's position. In the second, patient plans have been rigidly transposed on the phantom for patient-specific QA. All treatment plans have been generated using Multiplan (Accuray Inc., Sunnyvale, CA, US) with the dose calculation method RTrac (Ray Tracing). RTrac adopts a classical semianalytic method using experimental data such as off-axis ratio, TMR and output factors to calculate the dose kernel and the effective path length to correct for heterogeneities. The alternative calculation method adopts Monte Carlo and the definition of a virtual source (a validated and commissioned phase space file of the linac head is required) to calculate the dose. Although the MC method is more accurate in presence of heterogeneities such as in lung cancer treatments, it has minimal impact on brain treatment plans and is more time consuming, therefore the RTrac method has been adopted for this work [32].

CyberKnife ${ }^{\circledR}$ employs a multiple, non-isocentric, non-coplanar beam combination to produce optimized dose distributions in a predefined target. The treatment parameters and appropriate sequence of beamlets are optimized by the TPS according to user defined clinical objectives. A MU cutoff has been imposed to all plans to exclude beams with low MU, ensuring beam output stability.

Accurate beam delivery has been granted through the 6DSkull tracking system for real-time image guidance. This system acquires intra-fraction live images of the bony anatomy of patients' skull at userdefined intervals and compares them to the CT digitally reconstructed radiographs. The calculated compensation data are then sent to the robotic manipulator and used to compensate movements of the patient's head during treatment. In this way, the treatment beams can follow the tumor with sub millimetric precision. All plans have been delivered using the $5 \mathrm{~mm}$ diameter fixed collimator.

\section{Dose escalating plan and patient plans selection}

In order to assess the performance of the edgeless diodes, four plans are selected: three functional patient plans and one phantom plan (Rando-plans) with progressively escalated doses of 75, 100, 140 and 200 Gy at the Planning Target Volume (PTV). The Rando-plans are designed to evaluate the performance of the detectors in measuring dosimetric extreme conditions with strong dose gradients and high dose delivered (up to $200 \mathrm{~Gy}$ ) to a very small target size. The functional patient plans are real plans transposed on the RANDO phantom preserving beam ballistics and MUs of the clinical treatment. In both scenarios three 
edgeless diodes are placed respectively in correspondence of the centre of the PTV (position B - green circle ROI), at $+1 \mathrm{~cm}$ cranial direction (position $\mathrm{A}$ - orange circle ROI) and approximately $+2 \mathrm{~cm}$ caudal direction (position $\mathrm{C}$ - red circle ROI) as shown in Fig. 1. Differently from the other two probes, the diode in position $\mathrm{B}$ has been inserted along the cranio-caudal direction in the available phantom cavity. Table 1 summarises the main parameters of all plans calculated in the RANDO phantom, while Fig. 1 shows the positions of the detectors inside the phantom, respectively.

The correlation between accuracy of the dose measured by the edgeless diode and the number of directions of the incident beams has been recorded to evaluate if directionality may be still responsible for disagreements of the experimental data with the patient plans. The number of nodes (set of beamlets delivered from a unique position in space) in plans 1,2 and 3 are reported in Table 1 . The mean dose delivered to the target is approximately between 70 and $93 \mathrm{~Gy}$ and uncorrelated with the number of nodes of the treatment.

\section{CCO1 measurement on the EasyCube phantom}

The ionisation chamber CC01 is inserted in the central adapter of a EasyCube phantom (Euromechanics MedicalGmbH, Schwarzenbruck, Germany) and irradiated with the same three functional plans (plan 1, 2 and 3) that were delivered to the edgeless detectors. The choice of the EasyCube phantom for the CCO1 measurements is due to the
A
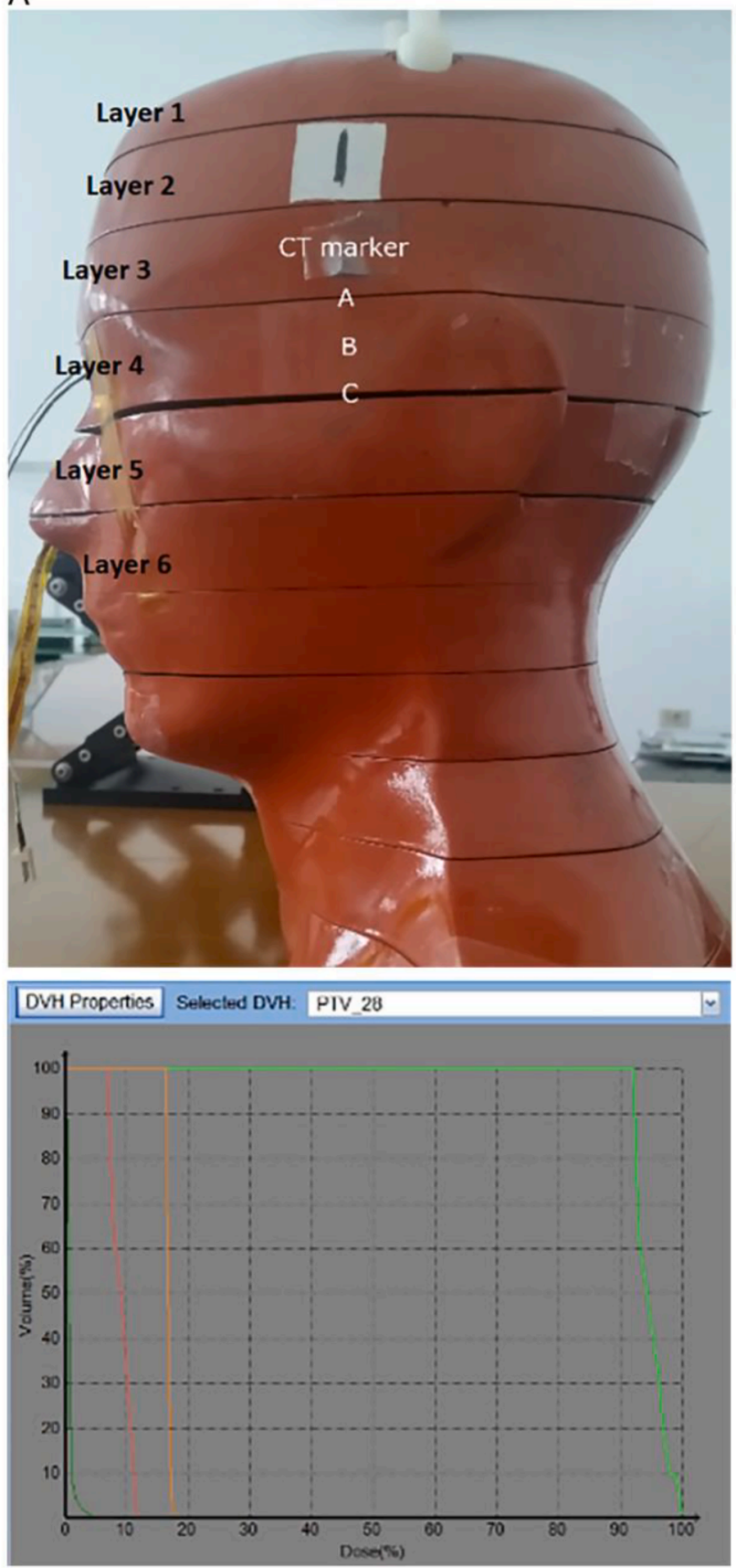

C
B
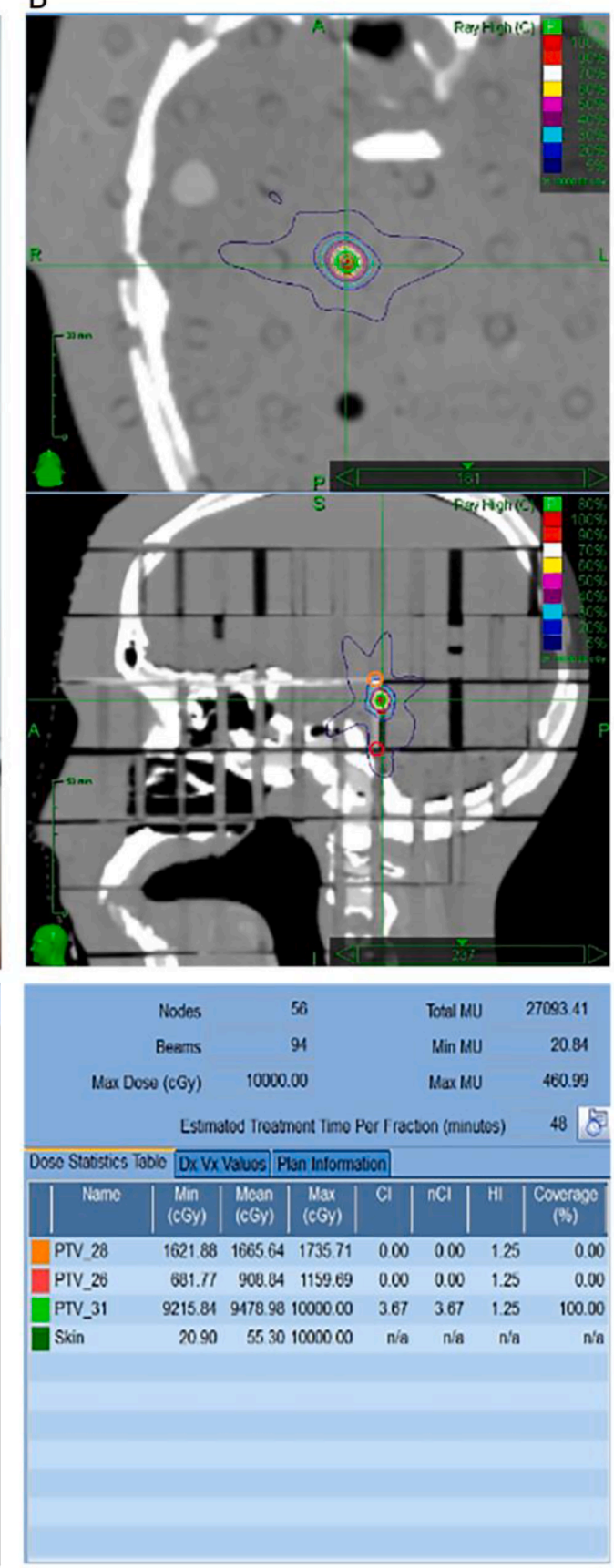

D

Fig. 1. a) Lateral view of the RANDO phantom assembled with the probes A and C between layer 3-4 and 4-5, respectively. Probe B is inserted in the hole adjacent to position A and fixed inside of layer 4 at a distance of $1 \mathrm{~cm}$ and $2 \mathrm{~cm}$ from probe A and C, respectively. b-d) Example of TPS plan with the regions of interest (ROI) corresponding to the detector locations. 
Table 1

Summary of the parameters of each patient plan.

\begin{tabular}{|c|c|c|c|c|c|c|c|c|c|c|c|}
\hline \multirow[t]{3}{*}{ Plan no. } & \multirow[t]{3}{*}{ No. of nodes } & \multirow[t]{3}{*}{ No. of beams } & \multicolumn{9}{|c|}{ TPS dose at each detector location } \\
\hline & & & \multicolumn{3}{|c|}{ Cranial (A) - (Gy) } & \multicolumn{3}{|c|}{ Target (B) - (Gy) } & \multicolumn{3}{|c|}{ Caudal (C) - (Gy) } \\
\hline & & & min & mean & $\max$ & $\min$ & mean & $\max$ & $\min$ & mean & $\max$ \\
\hline \multicolumn{12}{|c|}{ Real patient plans simulated on the RANDO phantom } \\
\hline 1 (patient) & 88 & 170 & 3.5 & 4.9 & 6.6 & 75.3 & 85.6 & 93.0 & 2.8 & 3.6 & 4.3 \\
\hline 2 (patient) & 93 & 161 & 9.4 & 11.1 & 14.0 & 86.5 & 92.4 & 98.3 & 2.2 & 3.0 & 3.8 \\
\hline 3 (patient) & 96 & 198 & 8.4 & 9.8 & 11.9 & 71.5 & 74.6 & 78.9 & 1.8 & 2.3 & 2.9 \\
\hline \multicolumn{12}{|c|}{ Plans calculated and simulated for the RANDO phantom } \\
\hline Rando 75 & 56 & 94 & 5.1 & 6.8 & 8.7 & 69.11 & 71.1 & 75.0 & 12.1 & 12.5 & 13.0 \\
\hline Rando 100 & 56 & 94 & 6.8 & 9.1 & 11.6 & 92.1 & 94.8 & 100.0 & 16.2 & 16.7 & 17.3 \\
\hline Rando 140 & 56 & 94 & 9.5 & 12.7 & 16.2 & 129.0 & 132.7 & 140.0 & 22.7 & 23.3 & 24.3 \\
\hline Rando 200 & 56 & 94 & 13.6 & 18.2 & 23.2 & 184.3 & 189.6 & 200.0 & 32.4 & 33.3 & 34.7 \\
\hline
\end{tabular}

impossibility of inserting the chamber within the RANDO without major mechanical modifications of the phantom. As with the edgeless detectors, the EasyCube phantom was CT imaged while the chamber was inserted within the phantom to estimate the dose at the exact location.

\section{Results}

\section{Dose rate dependence}

DPP measured on the samples used for the functional brain treatment irradiations and presented in this work, is shown in Fig. 2 along with the effect of pre-irradiation by a Cobalt- 60 gamma source to a total ionising dose of $40 \mathrm{kGy}$. The accumulated dose of $40 \mathrm{kGy}$ varies the sensitivity of the pre-irradiated diodes reducing their DPP variation within $5 \%$ of the reference conditions. The detectors overestimate the dose by approximately $10 \%$ at very low DPP (approx. $2.4 \times 10^{-5} \mathrm{~Gy} /$ pulse) corresponding to a few centimeters off axis. On the other hand, a not preirradiated diode shows very high sensitivity over the entire DPP range with over responses varying between $31 \%$ and $66 \%$ for dose per pulse of $2 \times 10^{-4}$ and $2.4 \times 10^{-5} \mathrm{~Gy} /$ pulse, respectively. In this work, pre-irradiated diodes were adopted to avoid the application of a correction factor to compensate for dose rate dependence.

\section{Linearity and calibration}

Dose linearity of the edgeless diodes shows an adjusted regression coefficient $\mathrm{R}^{2}$ of 0.999 and two standard deviation confidence of approximately $0.2 \%$. From the slope of the linear fit, the conversion

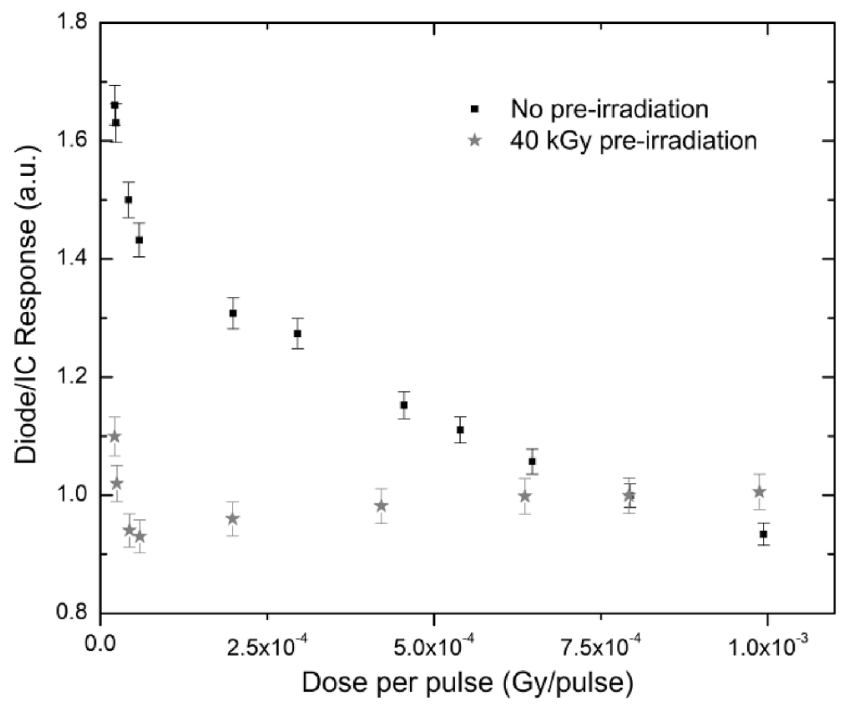

Fig. 2. DPP of preirradiated vs non irradiated edgeless diodes, measured as ratio of the diode's response to the response of a Farmer ion chamber (type 30013-PTW). factor has been extracted for all samples and is approximately $134 \pm 0.3$ $\mathrm{pC} / \mathrm{cGy}$.

\section{Output factors}

Fig. 3 shows the output factor of different cone field sizes ( 5 to 60 mm diameter) measured by the edgeless and PTW 60018 diodes at SAD of $800 \mathrm{~mm}$. Corresponding field correction factors reported by Francescon et al. [31] are applied to the data collected by the PTW 60018 to correct the over-response at small field sizes. No correction factors have been applied to the edgeless detectors which show an agreement with the reference detector within $\pm 0.51 \%$ in the field size range of 15 to 60 $\mathrm{mm}$. At smaller field sizes from 5 to $15 \mathrm{~mm}$ discrepancies do not exceed $\pm 1.6 \%$.

\section{Off-Axis ratio}

Fig. 4 shows the beam profiles acquired by the edgeless diode and compared to PTW 60018 diode. The profile data were obtained using cone collimators of four different circular field sizes (5, 10, 30 and 60 $\mathrm{mm}$ diameter), measured at depth of $15 \mathrm{~mm}$ and SAD of $800 \mathrm{~mm}$. The off-axis measurements were normalized to the central axis response for both the data sets. Table 2 shows Full Width Half Maximum (FWHM) and penumbra width $(80 \%-20 \%)$ of the beam profiles calculated using an interpolation-shape preserving fit (with a resolution step of 0.01 $\mathrm{mm}$ ).

The FWHM between the edgeless and stereotactic diodes agree within $2.5 \%$ for all the field sizes. The discrepancy in the penumbra width is within $0.06 \mathrm{~mm}$ excluding the fixed cone of $60 \mathrm{~mm}$ diameter which shows a discrepancy of $-0.21 \mathrm{~mm}$.

\section{Tissue-phantom ratio (TPR)}

Fig. 5 shows the comparison of TPR between the edgeless and PTW

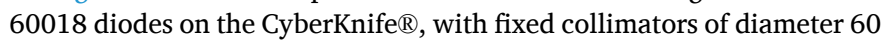
$\mathrm{mm}$. All measurements were performed in a large size water phantom at depths up to $20 \mathrm{~cm}$. Edgeless diode data agree with the PTW 60018 diode measurements within $2 \%$ for all depths except for the surface where the discrepancy is approximately $17 \%$. This is due to the build-up created by the packaging of the PTW 60018 which has a minimum thickness of $1 \mathrm{~mm}$ of solid water while the edgeless diode is packaged with only $0.07 \mathrm{~mm}$ of water equivalent material above the sensitive volume.

Table 3 summarises the beam parameters and dose linearity measured by edgeless diode and compared with the reference dosimeter (PTW 60018) adopted for commissioning the TPS. It is clearly seen that the dosimetric parameters of the CyberKnife ${ }^{\circledR}$ beam measured by the edgeless diodes agree with the clinical reference detector and are within the experimental uncertainties. This confirms the capability of the sensor to replicate the reference data for the TPS commissioning. The calibration factor is within $5 \%$ with data obtained in previous experiments with different generation machines and different diodes [23] 

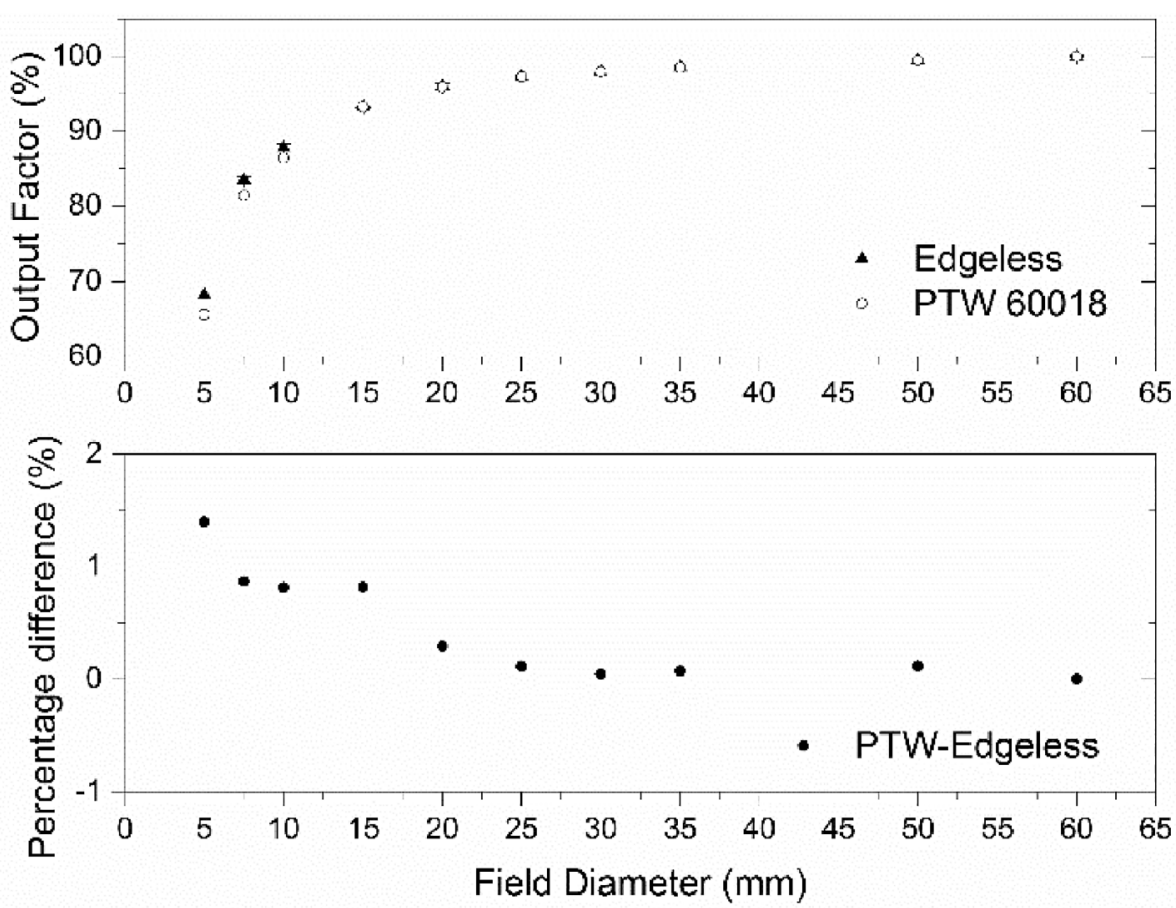

Fig. 3. Output factor for edgeless and PTW 60018 for fixed circular fields (5 to $60 \mathrm{~mm}$ ) for CyberKnife ${ }^{\circledR}$ at $\mathrm{SAD}$ of $800 \mathrm{~mm}$.

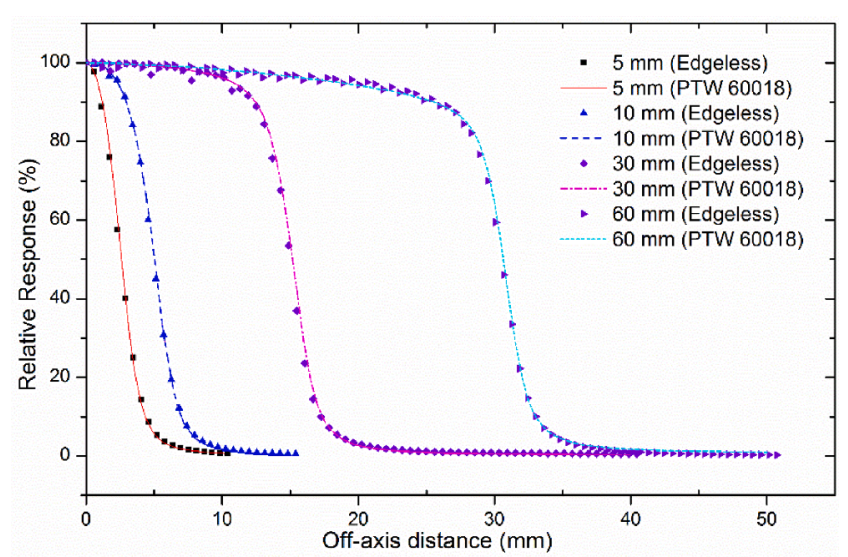

Fig. 4. Off-axis ratios measured by edgeless and PTW 60018 diodes on a CyberKnife ${ }^{\circledR}$ and fixed collimators of $5,10,30$ and $60 \mathrm{~mm}$, at a depth of $15 \mathrm{~mm}$ in a solid water phantom.

confirming the reproducibility and reliability of the diode's response and stability of the fabrication process.

\section{RANDO optimized and patient specific dose verification}

Table 4 shows the data obtained by the edgeless diodes in the anthropomorphic phantom for the patient and escalated treatment plans. Each row reports minimum, average and maximum values of the absorbed dose calculated by the TPS within a ROI of $1 \mathrm{~mm}$ diameter (defined around the position of each detector). The centre of the PTV matches the detector in position B (Table 4).

The column "mean-min and max-mean (\%)" represents the variation in percentage of the absorbed dose calculated by the TPS within the ROI. These values are used to evaluate the range of variation expected by the calculations and the agreement with the measurements. Tables 5 and 6 show the comparison between TPS and edgeless diodes in the cranial and caudal positions relative to the target, respectively.

Table 7 shows the absorbed dose calculated by TPS and measured by the ionisation chamber CC01 for the patients 1, 2 and 3 in the EasyCube phantom. The experiment could not be performed in the anthropomorphic phantom because the ionisation chamber could not be accommodated inside the RANDO without major mechanical modifications. The measurements obtained with the ionisation chamber aim to evaluate if there are significant effects of the volume size and angular dependence of the ionisation chamber in measuring patient specific QA for CyberKnife ${ }^{\circledR}$.

\section{Discussion}

The clinical and technological framework within which SRS is delivered has created new challenges for cancer care facilities quality assurance programs. Errors in planning or delivery for this kind of treatments are difficult or impossible to mitigate in subsequent sessions and dose verification should provide confidence not only in the magnitude of the delivered dose but also in its spatial distribution. The difficulties of this task are exacerbated by a lack of efficient and accurate

Table 2

Experimental results of FWHM and 20\%-80\% penumbra of both edgeless and PTW 60018 diodes, measured in CyberKnife G4, using fixed collimators.

\begin{tabular}{|c|c|c|c|c|c|c|c|c|}
\hline \multirow[b]{2}{*}{ Field size (mm) } & \multicolumn{2}{|l|}{ PTW 60018} & \multicolumn{2}{|l|}{ Edgeless } & \multicolumn{4}{|c|}{ PTW 60018-Edgeless difference } \\
\hline & FWHM (mm) & Penumbra (mm) & FWHM (mm) & Penumbra (mm) & $\Delta$ FWHM (\%) & $\Delta \mathrm{FWHM}(\mathrm{mm})$ & $\Delta$ Penumbra (\%) & $\Delta$ Penumbra $(\mathrm{mm})$ \\
\hline 5 & 5.23 & 1.99 & 5.09 & 2.02 & 2.57 & 0.14 & -1.56 & -0.03 \\
\hline 10 & 10.08 & 2.53 & 9.88 & 2.57 & 1.94 & 0.20 & -1.48 & -0.04 \\
\hline 30 & 30.34 & 2.83 & 29.80 & 2.89 & 1.77 & 0.54 & -2.26 & -0.06 \\
\hline 60 & 61.29 & 3.24 & 60.49 & 3.45 & 1.31 & 0.80 & -6.63 & -0.21 \\
\hline
\end{tabular}



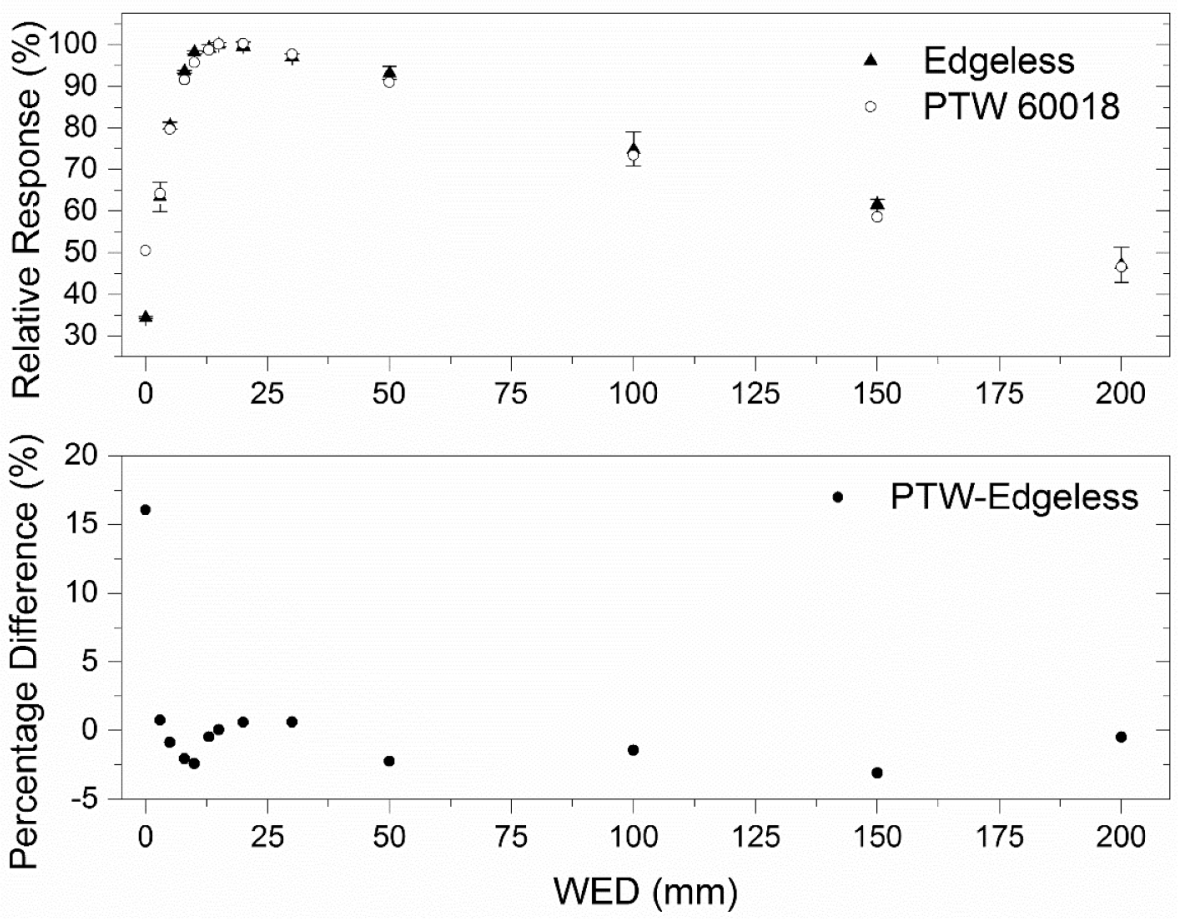

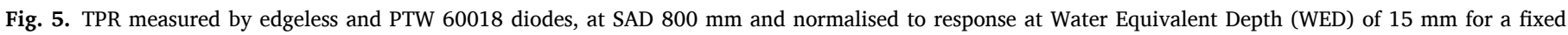
circular cone of $60 \mathrm{~mm}$ in diameter.

Table 3

Summary of the beam parameters and dose linearity data obtained by edgeless diodes.

\begin{tabular}{|c|c|c|c|}
\hline $\begin{array}{l}\text { Beam } \\
\text { parameter }\end{array}$ & $\begin{array}{l}\text { Range of the data } \\
\text { (Field Size }-\mathrm{cm} \text { ) }\end{array}$ & $\begin{array}{l}\text { Statistical } \\
\text { uncertainty (\%SD) }\end{array}$ & $\begin{array}{l}\text { Maximum } \\
\text { deviation }\end{array}$ \\
\hline $\begin{array}{l}\text { Output } \\
\text { factors }\end{array}$ & $0.5-6.0$ & 0.26 & $\begin{array}{l}\text { Field Size } 6.0-1.5 \\
\mathrm{~cm}: 0.12 \% \\
\text { Field Size } 1.5-0.5 \\
\mathrm{~cm}: 2.6 \%\end{array}$ \\
\hline TMR & $0-20 \mathrm{~cm}$ (depth) & 0.31 & $\begin{array}{l}\text { Depth } 0 \mathrm{~mm}: 16.1 \% \\
\text { Depth }>0 \mathrm{~mm}: 3 \%\end{array}$ \\
\hline $\begin{array}{r}\text { Off-axis } \\
\text { ratios }\end{array}$ & $\begin{array}{l}0.5-6 \mathrm{~cm} \text { (field } \\
\text { size) }\end{array}$ & 0.52 & $\begin{array}{l}\text { FWHM: } 2.6 \% \\
\text { Penumbra: } 0.21 \\
\text { mm }\end{array}$ \\
\hline $\begin{array}{l}\text { Dose } \\
\text { linearity }\end{array}$ & $100-400 \mathrm{cGy}$ & 0.3 & $\begin{array}{l}\text { Calibration factor: } \\
134 \mathrm{pC} / \mathrm{cGy}\end{array}$ \\
\hline
\end{tabular}

equipment and the measurement of patient specific dose delivered to a phantom represents a major challenge for any commercially available dosimeter. The measurement of three patient plans delivered to the EasyCube phantom equipped with a CC01 ionisation chamber shows a discrepancy of a maximum of -11.8 . \% when compared to the TPS calculation. The disagreement is due to two major limitations of the
CC01 for dosimetry in stereotactic radiosurgery for brain functional disorders: first, the volume averaging effect due to the very small field size at the target which makes the ionisation chamber under-responding systematically in all patients [31]. Additionally, there is an effect of angular dependence of the detector response [33]. This effect produces an increasing discrepancy between TPS and measurements proportional to the complexity of the plan, estimated by using the number of nodes used to deliver the dose. The correlation between discrepancy of the measurement and complexity of the plan for the three patients is approximately 0.997 indicating that there is a possibility that angular response dependence of the $\mathrm{CC} 01$ affects the accuracy in measuring dose in a non-isocentric treatment modality. A systematic evaluation of the performance of the CC01 in non-coplanar treatment plans for verification of stereotactic patient QA shows similar results with discrepancies in respect to the TPS up to $-15 \%$ [34].

Table 4 shows that the edgeless diode positioned within the PTV of the plans can measure the dose within $3.6 \%$ for all the patients without an evident correlation between discrepancy, dose delivered, or complexity of the plans. The diodes placed outside the PTV (hence not a target for treatment beams), in position A and C, show discrepancy within 1 Gy for all measurements. Excluding the plan Rando140 and Rando200 in the cranial position, where the diodes measure a discrepancy of -2.6 and $-4 \mathrm{~Gy}$ in respect to the TPS calculations, respectively.

Table 4

Comparison of data obtained by TPS calculations and Edgeless diodes at the target (position B) of the RANDO phantom.

\begin{tabular}{|c|c|c|c|c|c|c|c|c|}
\hline \multirow[t]{2}{*}{ Plan } & \multirow{2}{*}{$\begin{array}{l}\text { Dose prescription } \\
\text { (Gy) }\end{array}$} & \multicolumn{4}{|c|}{ TPS dose at target position B } & \multirow{2}{*}{$\begin{array}{l}\text { Edgeless dose at target position } \\
\text { B } \\
\text { mean } \pm 0.2 \mathrm{~Gy}\end{array}$} & \multirow{2}{*}{$\begin{array}{l}\Delta \text { [TPS-Edgeless] } \\
\text { (Gy) }\end{array}$} & \multirow[t]{2}{*}{$\Delta(\%)$} \\
\hline & & $\min (\mathrm{Gy})$ & mean (Gy) & $\max (\mathrm{Gy})$ & $\begin{array}{l}\text { \{mean-min ; max-mean }\} \\
(\%)\end{array}$ & & & \\
\hline 1 & 90 & 75.3 & 85.6 & 93.0 & $12.0 ; 8.6$ & 83.3 & 2.3 & 2.7 \\
\hline 2 & 90 & 86.5 & 92.4 & 98.3 & $6.3 ; 6.3$ & 94.9 & -2.5 & -2.7 \\
\hline 3 & 75 & 71.5 & 74.6 & 78.9 & $4.1 ; 5.7$ & 73.3 & 1.3 & 1.7 \\
\hline Rando 75 & 75 & 69.11 & 71.1 & 75.0 & $2.8 ; 5.4$ & 71.0 & 0.1 & 0.1 \\
\hline Rando 100 & 100 & 92.1 & 94.8 & 100.0 & $2.8 ; 5.4$ & 92.1 & 2.7 & 2.8 \\
\hline Rando 140 & 140 & 129.0 & 132.7 & 140.0 & $2.8 ; 5.5$ & 130.8 & 1.9 & 1.4 \\
\hline Rando 200 & 200 & 184.3 & 189.6 & 200.0 & $2.8 ; 5.5$ & 182.7 & 6.9 & 3.6 \\
\hline
\end{tabular}


Table 5

Comparison of data obtained by TPS calculations and Edgeless measurements at the cranial position A of the RANDO phantom.

\begin{tabular}{|c|c|c|c|c|c|c|c|}
\hline \multirow[t]{2}{*}{ Plan } & \multicolumn{4}{|c|}{ TPS dose at cranial position A } & \multirow{2}{*}{$\begin{array}{l}\text { Edgeless dose at cranial position A } \\
\text { mean } \pm 0.2 \text { Gy }\end{array}$} & \multirow[t]{2}{*}{$\Delta$ [TPS-Edgeless] (Gy) } & \multirow[t]{2}{*}{$\Delta(\%)$} \\
\hline & $\min (\mathrm{Gy})$ & mean (Gy) & $\max (\mathrm{Gy})$ & $\{$ mean-min ; max-mean $\}(\%)$ & & & \\
\hline 1 & 3.5 & 4.9 & 6.6 & $28.5 ; 34.6$ & 4.6 & 0.3 & 6.1 \\
\hline 2 & 9.4 & 11.1 & 14.0 & $15.3 ; 26.1$ & 11.3 & -0.2 & -1.8 \\
\hline 3 & 8.4 & 9.8 & 11.9 & $14.2 ; 21.4$ & 9.5 & 0.3 & 3.0 \\
\hline Rando 75 & 5.1 & 6.8 & 8.7 & $25.0 ; 27.9$ & 5.9 & 0.9 & 13.2 \\
\hline Rando 100 & 6.8 & 9.1 & 11.6 & $25.2 ; 27.4$ & 7.9 & 1.2 & 13.1 \\
\hline Rando 140 & 9.5 & 12.7 & 16.2 & $25.2 ; 27.5$ & 10.1 & 2.6 & 20.4 \\
\hline Rando 200 & 13.6 & 18.2 & 23.2 & $25.2 ; 27.4$ & 14.0 & 4.2 & 23.0 \\
\hline
\end{tabular}

Table 6

Comparison of data obtained by TPS calculations and Edgeless measurements at the caudal position C of the RANDO phantom.

\begin{tabular}{|c|c|c|c|c|c|c|c|}
\hline \multirow[t]{2}{*}{ Plan } & \multicolumn{4}{|c|}{ TPS dose at caudal position C } & \multirow{2}{*}{$\begin{array}{l}\text { Edgeless dose at caudal position } C \\
\text { mean } \pm 0.2 \text { Gy }\end{array}$} & \multirow[t]{2}{*}{$\Delta$ [TPS-Edgeless] (Gy) } & \multirow[t]{2}{*}{$\Delta(\%)$} \\
\hline & $\min (\mathrm{Gy})$ & mean (Gy) & $\max (\mathrm{Gy})$ & \{mean-min; max-mean\} (\%) & & & \\
\hline 1 & 2.8 & 3.6 & 4.3 & $22.2 ; 19.4$ & 3.5 & 0.1 & 2.7 \\
\hline 2 & 2.2 & 3.0 & 3.8 & $26.6 ; 26.6$ & 2.6 & 0.4 & 13.3 \\
\hline 3 & 1.8 & 2.3 & 2.9 & $21.7 ; 26.0$ & 2.1 & 0.2 & 8.7 \\
\hline Rando 75 & 12.1 & 12.5 & 13.0 & $3.2 ; 4.0$ & 11.9 & 0.6 & 4.8 \\
\hline Rando 100 & 16.2 & 16.7 & 17.3 & $3.0 ; 3.6$ & 15.7 & 1 & 5.9 \\
\hline Rando 140 & 22.7 & 23.3 & 24.3 & $2.5 ; 4.3$ & 19.3 & 4 & 17.1 \\
\hline Rando 200 & 32.4 & 33.3 & 34.7 & $2.7 ; 4.2$ & 32.9 & 0.4 & 1.2 \\
\hline
\end{tabular}

Table 7

Comparison of data obtained by TPS calculations and ionisation chamber CC01 in the target position using the EasyCube phantom.

\begin{tabular}{|c|c|c|c|c|c|c|c|}
\hline \multirow[t]{2}{*}{ Plan } & \multicolumn{4}{|c|}{ TPS dose at target of EasyCube phantom } & \multirow{2}{*}{$\begin{array}{l}\text { CC01 } \\
\text { at } \\
\text { target } \\
\text { mean } \\
\pm 0.1 \\
\text { Gy }\end{array}$} & \multirow{2}{*}{$\begin{array}{l}\Delta \text { [TPS- } \\
\text { CC01] } \\
\text { (Gy) }\end{array}$} & \multirow{2}{*}{$\begin{array}{l}\Delta \\
(\%)\end{array}$} \\
\hline & $\begin{array}{l}\min \\
\text { (Gy) }\end{array}$ & $\begin{array}{l}\text { mean } \\
\text { (Gy) }\end{array}$ & $\begin{array}{l}\max \\
\text { (Gy) }\end{array}$ & $\begin{array}{l}\{\text { mean- } \\
\text { min; max- } \\
\text { mean }\}(\%)\end{array}$ & & & \\
\hline 1 & 74.14 & 84.73 & 92.65 & $12.5 ; 9.3$ & 79.51 & 5.22 & 6.1 \\
\hline 2 & 81.80 & 86.60 & 92.30 & $5.5 ; 6.6$ & 78.50 & 8.10 & 9.3 \\
\hline 3 & 67.74 & 70.80 & 75.30 & $4.3 ; 6.3$ & 62.46 & 8.34 & 11.8 \\
\hline
\end{tabular}

Although this disagreement seems large, it is well within the range of uncertainty calculated by the TPS within the ROI of $1 \mathrm{~mm}$ diameter defined in the plan and positioned around the position of the sensors, which varies from 6.7 Gy and 9.6 Gy for Rando140 and Rando200, respectively.

The same disagreement pattern between TPS and diodes is found in the caudal position for the plan Rando140 and Rando200. The overall discrepancy is within approximately 4 Gy over 24 Gy estimated by the TPS with a percentage difference of $17 \%$. This may suggest a slight misalignment between TPS ROIs and actual position of the detectors in this very high dose gradient region.

\section{Conclusions}

Stereotactic Radiosurgery using the CyberKnife ${ }^{\circledR}$ system is one of the options for treatment of functional neurological disorders. The high doses delivered to the patient and the proximity of sensitive areas of the brain such as the brainstem, would benefit from a QA procedure that guarantee accuracy, precision and confidence in the clinicians. QA of such treatments is limited to machine operational verification as there is no commercial instrumentation able to measure simultaneously high doses absorbed in a small field using non-isocentric robotic delivery. In this work we propose the edgeless diodes as a solution to fill the technological gap between complexity of the treatment delivery and instrumentation available for quality control. The edgeless diodes are manufactured and packaged using a unique technology which minimises the beam perturbation and angular dependence of the detector response. The diodes show agreement within experimental uncertainties with state-of-the-art reference dosimeters for machine QA and small field dosimetry as confirmed by the measurements of output factor, offaxis and tissue-phantom ratios for fixed cone from 5 to $60 \mathrm{~mm}$. In order to evaluate the performance of such detectors in patient specific QA of functional treatments, three patients and a set of dose escalated plans have been measured on the anthropomorphic phantom RANDO with three diodes, one placed within the PTV and the other two in adjacent healthy tissue. The dose measured by edgeless detectors agree with the TPS within $3.6 \%$ in the PTV for the all set of plans. The same experiment performed on the same patient plans using a CC01 shows discrepancy up to $-11.8 \%$. The edgeless technology represents a viable solution for small field dosimetry in non-isocentric robotic and non-coplanar delivery modalities and could be implemented in other advanced radiotherapy treatments. The technology allows also the fabrication of pixelated arrays for high resolution $2 \mathrm{D}$ or $3 \mathrm{D}$ dose mapping.

\section{Declaration of Competing Interest}

The authors declare that they have no known competing financial interests or personal relationships that could have appeared to influence the work reported in this paper.

\section{Acknowledgments}

S. Alhujaili would like to thank Aljouf University for their support.

This research did not receive any specific grant from funding agencies in the public, commercial, or not-for-profit sectors.

\section{References}

[1] Friehs GM, Park MC, Goldman MA, Zerris VA, Norén G, Sampath P. Stereotactic radiosurgery for functional disorders. Neurosurg Focus 2007;23(6):E2. https://doi. org/10.3171/FOC-07/12/E3.

[2] Kondziolka D. In: Textbook of Stereotactic and Functional Neurosurgery. Berlin Heidelberg: Springer-Verlag; 2009. p. 1191-202.

[3] Dieterich S, Cavedon C, Chuang CF, Cohen AB, Garrett JA, Lee CL, et al. Report of AAPM TG 135: Quality assurance for robotic radiosurgery. Med Phys 2011;38 (6Part1):2914-36. https://doi.org/10.1118/1.3579139.

[4] IAEA. Dosimetry of Small Static Fields Used in External Beam Radiotherapy. Vienna: IAEA; 2017.

[5] ICRU. ICRU Report 91 - Prescribing, Recording, and Reporting of Stereotactic Treatments with Small Photon Beams, 2014. 
[6] Taylor ML, Kron T, Franich RD. Acta Oncologica A contemporary review of stereotactic radiotherapy: Inherent dosimetric complexities and the potential for detriment 2011. https://doi.org/10.3109/0284186X.2010.551665.

[7] Kairn T, Charles P, Crowe SB, Trapp JV. Effects of inaccurate small field dose measurements on calculated treatment doses. Australas Phys Eng Sci Med 2016;39 (3):747-53. https://doi.org/10.1007/s13246-016-0461-y.

[8] Kilby W, Dooley JR, Kuduvalli G, Sayeh S, Maurer CR. The CyberKnife ${ }^{\circledR}$ robotic radiosurgery system in 2010. Technol Cancer Res Treat 2010;9(5):433-52. https:// doi.org/10.1177/153303461000900502.

[9] Pappas E, Maris TG, Zacharopoulou F, Papadakis A, Manolopoulos S, Green S, et al. Small SRS photon field profile dosimetry performed using a PinPoint air ion chamber, a diamond detector, a novel silicon-diode array (DOSI), and polymer gel dosimetry Analysis and intercomparison. Med Phys 2008;35(10):4640-8. https:// doi.org/10.1118/1.2977829.

[10] Tyler M, Liu PZY, Chan KW, Ralston A, McKenzie DR, Downes S, et al. Characterization of small-field stereotactic radiosurgery beams with modern detectors. Phys Med Biol 2013;58(21):7595-608. https://doi.org/10.1088/00319155/58/21/7595.

[11] Wilcox EE, Daskalov GM. Evaluation of GAFCHROMIC® EBT film for CyberKnife ${ }_{\circledR}$ dosimetry. Med Phys 2007;34(6 Part1):1967-74. https://doi.org/10.1118/ 1.2734384 .

[12] Blanck O, Masi L, Damme M-C, Hildebrandt G, Dunst J, Siebert F-A, et al. Filmbased delivery quality assurance for robotic radiosurgery: Commissioning and validation. Phys Medica 2015;31(5):476-83. https://doi.org/10.1016/j. ejmp.2015.05.001.

[13] Devic S, Tomic N, Lewis D. Reference radiochromic film dosimetry: Review of technical aspects. Phys Medica 2016;32(4):541-56. https://doi.org/10.1016/j. ejmp.2016.02.008.

[14] Hardcastle N, Basavatia A, Bayliss A, Tomé WA. High dose per fraction dosimetry of small fields with Gafchromic EBT2 film. Med Phys 2011;38(7):4081-5. https:// doi.org/10.1118/1.3597834.

[15] Palmer AL, Dimitriadis A, Nisbet A, Clark CH. Evaluation of Gafchromic EBT-XD film, with comparison to EBT3 film, and application in high dose radiotherapy verification. Phys Med Biol 2015;60(22):8741-52. https://doi.org/10.1088/0031 9155/60/22/8741.

[16] Ashland. GAFCHROMIC EBT n.d. http://www.gafchromic.com/gafchromic-film/ radiotherapy-films/EBT/index.asp (accessed September 20, 2003).

[17] Cusumano D, Fumagalli ML, Marchetti M, Fariselli L, De Martin E. Dosimetric verification of stereotactic radiosurgery/stereotactic radiotherapy dose distributions using Gafchromic EBT3. Med Dosim 2015;40(3):226-31. https://doi org/10.1016/j.meddos.2015.01.001.

[18] Cusumano D, Fumagalli ML, Ghielmetti F, Rossi L, Grossi G, Lanzarotti R, et al. Sum signal dosimetry: A new approach for high dose quality assurance with Gafchromic EBT3. J Appl Clin Med Phys 2017;18(2):181-90. https://doi.org/ $10.1002 / \mathrm{acm} 2.12045$.

[19] Wong JHD, Knittel T, Downes S, Carolan M, Lerch MLF, Petasecca M, et al. The use of a silicon strip detector dose magnifying glass in stereotactic radiotherapy QA and dosimetry. Med Phys 2011;38(3):1226-38.
[20] Shi J, Simon WE, Zhu TC. Modeling the instantaneous dose rate dependence of radiation diode detectors. Med Phys 2003;30(9):2509-19. https://doi.org/ 10.1118/1.1602171.

[21] Benmakhlouf H, Andreo P. Spectral distribution of particle fluence in small field detectors and its implication on small field dosimetry. Med Phys 2017;44(2): 713-24. https://doi.org/10.1002/mp.12042.

[22] Andreo P. The physics of small megavoltage photon beam dosimetry. Radiother Oncol 2018;126(2):205-13. https://doi.org/10.1016/j.radonc.2017.11.001.

[23] Alhujaili SF, Biasi G, Alzorkany F, Grogan G, Al Kafi MA, Lane J, et al. Quality assurance of Cyberknife robotic stereotactic radiosurgery using an angularly independent silicon detector. J Appl Clin Med Phys 2019;20(1):76-88. https://doi. org/10.1002/acm2.2019.20.issue-110.1002/acm2.12496.

[24] Petasecca M, Alhujaili S, Aldosari AH, Fuduli I, Newall M, Porumb CS, et al. Angular independent silicon detector for dosimetry in external beam radiotherapy. Med Phys 2015;42(8):4708-18. https://doi.org/10.1118/1.4926778.

[25] Bouchard H, Seuntjens J, Duane S, Kamio Y, Palmans H. Detector dose response in megavoltage small photon beams I. Theoretical concepts. Med Phys 2015;42(10): 6033-47. https://doi.org/10.1118/1.4930053.

[26] Fuduli I, Porumb C, Espinoza AA, Aldosari AH, Carolan M, Lerch MLF, et al. A comparative analysis of multichannel Data Acquisition Systems for quality assurance in external beam radiation therapy. J Instrum 2014;9:T06003. https:// doi.org/10.1088/1748-0221/9/06/T06003.

[27] Mazza G, Cirio R, Donetti M, La Rosa A, Luparia A, Marchetto F, et al. A 64-channel wide dynamic range charge measurement ASIC for strip and pixel ionization detectors. IEEE Trans Nucl Sci 2005;52(4):847-53. https://doi.org/10.1109/ TNS.2005.852702.

[28] Shi J, Simon WE, Zhu TC, Modeling the instantaneous dose rate dependence of radiation diode detectors, Med Phys. 2003 Sep;30(9):2509-19; Saini AS, Zhu TC, Dose rate and SDD dependence of commercially available diode detectors Med Phys. 2004 Apr;31(4):914-24.

[29] Rikner G. Silcon diodes as detectors in relative dosimetry of photon, electron and proton radiation fields (PhD Thesis), iNSPIRE-HEP, 1983, ISBN: 9789155414481.

[30] Wang Y, Easterling SB, Ting JY. Ion recombination corrections of ionization chambers in flattening filter-free photon radiation. J Appl Clin Med Phys 2012;13 (5):262-8.

[31] Francescon P, Kilby W, Satariano N. Monte Carlo simulated correction factors for output factor measurement with the CyberKnife system - Results for new detectors and correction factor dependence on measurement distance and detector orientation. Phys Med Biol 2014;59:N11. https://doi.org/10.1088/0031-9155/59/ 6/N11.

[32] Murali V, Gopalakrishna Kurup PG, Bhuvaneswari N, Sudahar H, Muthukumaran M. Monte Carlo and ray tracing algorithms in the cyberknife treatment planning for lung tumours- comparison and validation. J Radiosurgery SBRT 2013;2:85-98.

[33] Feldman J, Orion I. Small Volume Ionization Chambers Angular Dependence and Its Influence on Point-Dose Measurements. Int J Med Physics, Clin Eng Radiat Oncol 2016;5:26-32. https://doi.org/10.4236/ijmpcero.2016.51003.

[34] Tas B, Faruk Durmus I. Dose Verification With Different Ion Chambers For FFF Energy Plans. Int J Eng Sci || n.d.:23-42. https://doi.org/10.9790/1813-07100 26669. 